\title{
Spot Pricing of Electricity and Ancillary Services in a Competitive California Market
}

\author{
Afzal S. Siddiqui, Chris Marnay, and Mark Khavkin \\ Environmental Energy Technologies Division \\ Ernest Orlando Lawrence Berkeley National Laboratory \\ Berkeley, California 94720
}

November 2000

Download from: http://eetd.lbl.gov/EA/EMP/

The work described in this study was funded by the Assistant Secretary of Energy Efficiency and Renewable Energy, Office of Power Technologies of the U.S. Department of Energy under Contract No. DE-AC03-76SF00098. 


\title{
Spot Pricing of Electricity and Ancillary Services in a Competitive California Market
}

\author{
Afzal S. Siddiqui, Chris Marnay, and Mark Khavkin \\ Environmental Energy Technologies Division \\ Ernest Orlando Lawrence Berkeley National Laboratory \\ Berkeley, California 94720 \\ email: ASSiddiqui@lbl.gov
}

\begin{abstract}
Typically, in competitive electricity markets, the vertically integrated utilities that were responsible for ensuring system reliability in their own service territories, or groups of territories, cease to exist. The burden falls to an independent system operator (ISO) to ensure that enough ancillary services (AS) are available for safe, stable, and reliable operation of the grid, typically defined, in part, as compliance with officially approved engineering specifications for minimum levels of $A S$. In order to characterize the behavior of market participants (generators, retailers, and an ISO) in a competitive electricity market with reliability requirements, spot markets for both electricity and AS are modeled. By assuming that each participant seeks to maximize its wealth and that all markets clear, we solve for the optimal quantities of electricity and AS traded in the spot market by all participants, as well as the market clearing prices for each.
\end{abstract}

Keywords: Ancillary services, competitive electricity markets, equilibrium pricing.

\section{Introduction}

Once thought of as a "natural monopoly" industry, i.e., one in which cost perpetually declines with output, thereby rendering competition infeasible, the electric power sector is now undergoing policy and regulatory changes intended to foster competition. In the U. S., the electric power industry has predominantly been vertically integrated with its various functions conglomerated under the auspices of an investor-owned utility company that holds an exclusive franchise to provide services to a certain geographic area. According to [1], the four main electricity supply functions provided by a utility are:

- generation: conversion of primary energy to electricity.
- transmission: transportation of electricity along meshed high-voltage wires to substations.

- distribution: transportation of electricity along low-voltage wires to customer meters.

- retailing: arrangements for billing and demand management.

California was among the first U. S. states to deregulate its electric power sector. Similar to electricity market reforms in other regions of the world, the changes in California's industry included unbundling the various services previously offered by its three major incumbent investor-owned electricity utilities. Now, instead of allowing these utilities to control all aspects of electricity supply, California state legislators passed Assembly Bill (AB) 1890 which separates the industry into:

1. a competitive part, consisting of the generation and retail functions, and

2. a regulated monopoly structure that retained control over the transmission and distribution systems.

Two non-profit corporations were created: the California Independent System Operator (CAISO) and the California Power Exchange (CalPX). The former provides system control to all electricity suppliers, while the latter operates forward competitive energy markets from which distribution companies must buy electricity for their retained retail customers for a transitional period, and into which generators sell. In addition, the CAISO manages a real-time imbalance energy market, which is essentially a spot market for wholesale electricity. AS are required under North American Electric Reliability Council (NERC) and Western System Coordinating Council (WSCC) rules to balance energy and safeguard the reliability of the grid. AS are also procured by the CAISO in competitive day- and hourahead markets. 
Each scheduling provider (SC) can choose to supply its own AS, and the Federal Energy Regulatory Commission (FERC) has approved a block forward AS market operated by CalPX. Nonetheless, to date, virtually all AS have been procured in CAISO's first in the world open competitive AS markets. That is, the CAISO accepts generator offers and buys AS on behalf of almost all loads. The AS procured in this way are:

- regulation service: generation that is available and running, and can be used to maintain realtime energy balance.

- spinning reserves: generation that is running and synchronous with additional capacity available.

- non-spinning reserves: generation that is available quickly but not running.

- replacement reserves: generation that is capable of starting up and running for a sustained period.

In addition, reactive power support and black-start generation capability are AS that are procured through annual contracts. For a more complete description of the restructured California electricity industry, see either [2] or [3].

Regardless of the form of deregulation, it has been documented that introduction of competition in the electricity generation sector leads to some improvements in social welfare, as wholesale prices tend to be lower and labor productivity increases (see [4]). Along with greater economic efficiency in the generation sector, however, deregulation has also introduced new problems into an industry that was once insulated from the forces of the free market. Some of these issues include:

- market power: some evidence exists that both the British and California wholesale electricity markets have at least the conditions that reward the withholding of generation capacity from the market (see [5], [6], and [7]).

- price volatility: although some price volatility is to be expected in any competitive commodity market due to supply and demand conditions, seasonality, and lack of storability, there has been evidence that some price volatility may have been caused or exacerbated by firms exercising market power (see either [2] or [8]).

- management of system reliability: the vertically integrated utilities operated in a strictly hierarchical manner with a central controller responsible for marshalling all the resources necessary to operate the system reliably. Particularly, AS were self-provided by utilities based on engineering specifications suggested by regional reliability councils. In restructured markets, their procurement is typically the duty of an independent system operator (ISO) which is responsible for the transmission grid.

While the first two issues have received considerable attention in the literature, the third one has not been addressed via an integrated model that acknowledges the fundamental link between the electricity and AS markets, viz., electricity and AS are substitute products, implying that the physical and financial characteristics of one market will have consequences for the other. Indeed, the problem of managing system reliability in a competitive environment may be exacerbated if there is no market-based methodology for pricing AS. It is with this issue in mind that spot markets for electricity and AS in a perfectly competitive framework are modeled here. Our objective in solving the model is to assess equilibrium prices for electricity and AS and to determine what factors will affect the trading decisions of the various agents in the markets. The structure of this paper is as follows:

- Section 2 introduces the model of electricity production and spot markets.

- Section 3 solves for the equilibrium prices in each market and discusses the intuition behind the resulting price structure.

- Section 4 compares the results from Section 3 to California market data.

- Section 5 summarizes the main results and gives direction for future research in this area.

\section{Electricity Production and Spot Markets}

Since our objective is to assess equilibrium spot prices for electricity and AS, we require a model of the spot markets and of the transactions conducted there. We assume perfectly competitive ${ }^{1}$ spot markets for electricity and one type of AS (as opposed to the four that actually exist in California). We analyze production decisions for a single future time period because the non-storability of electricity creates markets that are

\footnotetext{
${ }^{1}$ The degree to which the California electricity markets are competitive is open to debate. Our concern, however, is more with how to price AS once market mechanisms are fully in place.
} 
independent over time. For simplicity, we assume that there is no uncertainty in the spot markets. Underlying this assumption is the fact that power companies are able to forecast demand in the immediate future, i.e., the next hour, with precision. Here, we also abstract from transmission constraints by supposing that electricity can be transmitted costlessly. Of course, in reality transmission bottlenecks play a significant role in determining the pattern of electricity generation and pricing. However, our focus is on the short term strategies of market agents that will determine equilibrium spot prices rather than on congestion pricing.

Within this framework, we have three distinct types of agents who have various interests in the markets:

- $\mathbf{n} \in \mathcal{Z}_{+}$identical generators: generator $p_{i}$ has $\alpha_{p_{i}}$ megawatts (MW) of production capacity available for any given period. It can use this capacity either to generate electricity and sell it into the electricity spot market or to reserve the capacity and sell it into the AS spot market. For selling the output from $X_{p_{i}}$ MW of capacity into the electricity spot market, generator $p_{i}$ receives the endogenously-determined electricity spot price $P_{X}$. On the other hand, if it sells $Y_{p_{i}}$ MW of capacity into the AS spot market, the generator receives the endogenously-determined per MW AS spot price $P_{Y}$. In addition, if the generator is called upon to produce electricity from these $Y_{p_{i}}$ MW of capacity for any reason, it also receives the per MW electricity spot price $P_{X}$.

- $\mathbf{m} \in \mathcal{Z}_{+}$identical retailers: retailer $r_{j}$ purchases electricity from the spot market and sells it to customers in its exclusive franchise area at a fixed unit price of $P_{r_{j}}$. The total retail demand for electricity in its area, $X_{r_{j}}^{T}$, is known with certainty at the time of the decision to purchase and must be satisfied, i.e., the retailer faces an obligation to serve a totally inelastic demand in the short run. This setup reflects the fact that in California, most enduse consumers do not yet see volatile spot prices; rather, they are guaranteed fixed per unit prices. The retailer, however, has to take the risk of purchasing from a volatile market. This would seem to imply that retailers would like to purchase futures contracts to lock in their purchase prices. The issue of hedging is not addressed is here, but is given full treatment in [9].

- an ISO: the ISO procures enough AS from the spot market to comply with the minimum levels required for reliability. Usually in California, this implies that the amount of AS procured by the
ISO is approximately a fixed percentage of overall electricity demand. The ISO, thus, acquires a predictable amount of AS from the spot market.

As we shall show in Section 3, each type of agent acts out of self-interest in order to maximize its wealth. Of course, the decisions made by the retailers and the ISO are trivial since they have to purchase certain quantities of electricity and AS, respectively. The generator's problem, however, is more subtle in that it involves production of substitutes. The interaction of these agents in the markets then determines the equilibrium spot prices for electricity and AS.

\section{Spot Market Trading}

In order to determine the equilibrium spot prices for electricity and AS, we first set up the optimization problems of the market participants. Applying the notation and assumptions of Section 2, we can express the profit-maximization problem of generator $p_{i}$ :

$$
\begin{aligned}
\pi_{p_{i}}^{*}\left(X_{p_{i}}, Y_{p_{i}}\right)= & \max _{X_{p_{i}}, Y_{p_{i}}}\left\{P_{X} X_{p_{i}}+P_{Y} Y_{p_{i}}\right. \\
& +P_{X} f Y_{p_{i}} \\
& -\frac{\theta_{\ell}}{2 \alpha_{p_{i}}}\left(X_{p_{i}}+Y_{p_{i}}\right)^{2} \\
& \left.-\frac{\theta_{f}}{2 \alpha_{p_{i}}}\left(X_{p_{i}}+f Y_{p_{i}}\right)^{2}\right\}
\end{aligned}
$$

where $\pi_{p_{i}}^{*}$ is the maximized profit level, $\theta_{\ell}>0$ and $\theta_{f}>0$ are the per MW labor and fuel costs, respectively, and $0 \leq f \leq 1$ denotes the fraction of AS capacity sold that is called upon to generate. ${ }^{2}$ Labor costs are incurred for both electricity and AS since the generator needs to have enough labor available to meet all potential generation requirements. On the other hand, fuel cost is incurred only for actual electricity generation, i.e., to produce electricity sold as energy, and to operate any AS capacity that is specifically required by the ISO to generate. Furthermore, both cost terms exhibit quadratic forms, which implies increasing marginal costs of generation. Intuitively, this models the fact that as demand increases, less efficient sources of generation are brought on line. For the purposes of this model, we assume that continuous quadratic functions reasonably approximate generation costs, even though actual generation costs may be discontinuous.

The profit-maximization problem of retailer $r_{j}$ is as follows:

$$
\pi_{r_{j}}^{*}\left(X_{r_{j}}\right)=\max _{X_{r_{j}}}\left\{P_{r_{j}} X_{r_{j}}^{T}-P_{X} X_{r_{j}}\right\}
$$

\footnotetext{
${ }^{2}$ We assume that cost and AS call parameters are known with certainty to all market participants. Also, we assume that generators have sufficient capacity to meet system demand.
} 


$$
\text { subject to } X_{r_{j}} \geq X_{r_{j}}^{T}
$$

where $\pi_{r_{j}}^{*}$ is the maximized profit level, and $X_{r_{j}}^{T}$ is the realized total electricity demand in the franchise area of retailer $r_{j}$. Similarly, the ISO's optimization problem can be written as follows:

$$
\begin{aligned}
\pi_{I}^{*}\left(Y_{I}\right)= & \max _{Y_{I}}\left\{-P_{Y} Y_{I}\right\} \\
& \text { subject to } Y_{I} \geq Y_{I}^{T} \equiv \gamma X^{T}
\end{aligned}
$$

where $\pi_{I}^{*}$ is the maximized profit level, $Y_{I}$ is the amount of AS purchased by the ISO from the spot market, and $Y_{I}^{T}$ is its total purchase requirement. Note that the total amount of AS required equals some fraction $0 \leq$ $\gamma \leq 1$ of the overall electricity load $X^{T} \equiv \sum_{j=1}^{m} X_{r_{j}}^{T}$.

Generator $p_{i}$ 's real-time decision is to select the quantity of electricity and AS to sell into the spot markets that maximize its profits. The first-order necessary conditions are:

$$
\begin{array}{ll} 
& \frac{\partial \pi_{p_{i}}^{*}\left(X_{p_{i}}, Y_{p_{i}}\right)}{\partial X_{p_{i}}}=0 \\
\Rightarrow \quad & P_{X}-\frac{\theta_{\ell}}{\alpha_{p_{i}}}\left(X_{p_{i}}^{*}+Y_{p_{i}}^{*}\right) \\
& -\frac{\theta_{f}}{\alpha_{p_{i}}}\left(X_{p_{i}}^{*}+f Y_{p_{i}}^{*}\right)=0 \\
\Rightarrow \quad & X_{p_{i}}^{*}=\frac{\alpha_{p_{i}} P_{X}-\left(\theta_{\ell}+f \theta_{f}\right) Y_{p_{i}}^{*}}{\theta_{\ell}+\theta_{f}} \\
& \frac{\partial \pi_{p_{i}}^{*}\left(X_{p_{i}}, Y_{p_{i}}\right)}{\partial Y_{p_{i}}}=0 \\
\Rightarrow \quad & P_{Y}+f P_{X}-\frac{\theta_{\ell}}{\alpha_{p_{i}}}\left(X_{p_{i}}^{*}+Y_{p_{i}}^{*}\right) \\
& -\frac{\theta_{f}}{\alpha_{p_{i}}}\left(f X_{p_{i}}^{*}+f^{2} Y_{p_{i}}^{*}\right)=0 \\
& Y_{p_{i}}^{*}=\frac{\alpha_{p_{i}}\left(P_{Y}+f P_{X}\right)}{\theta_{\ell}+f^{2} \theta_{f}} \\
& -\frac{\left(\theta_{\ell}+f \theta_{f}\right) X_{p_{i}}^{*}}{\theta_{\ell}+f^{2} \theta_{f}}
\end{array}
$$

The second-order sufficiency conditions are also satisfied as the hessian matrix, $H_{\pi_{p_{i}}\left(X_{p_{i}}, Y_{p_{i}}\right)}$, is negative definite, i.e., the determinants of the principal minors are nonzero and alternate in sign with the first one being negative:

$$
\begin{gathered}
H_{\pi_{p_{i}}\left(X_{p_{i}}, Y_{p_{i}}\right)}=\left[\begin{array}{cc}
-\frac{\left(\theta_{\ell}+\theta_{f}\right)}{\alpha_{p_{i}}} & -\frac{\left(\theta_{\ell}+f \theta_{f}\right)}{\alpha_{p_{i}}} \\
-\frac{\left(\theta_{\ell}+f \theta_{f}\right)}{\alpha_{p_{i}}} & -\frac{\left(\theta_{\ell}+f^{\theta_{f}}\right)}{\alpha_{p_{i}}}
\end{array}\right] \\
\Rightarrow \operatorname{det}\left(-\frac{\left(\theta_{\ell}+\theta_{f}\right)}{\alpha_{p_{i}}}\right)=-\frac{\left(\theta_{\ell}+\theta_{f}\right)}{\alpha_{p_{i}}}<0
\end{gathered}
$$

and

$$
\operatorname{det}\left(H_{\pi_{p_{i}}\left(X_{p_{i}}, Y_{p_{i}}\right)}\right)=\frac{(1-f)^{2} \theta_{\ell} \theta_{f}}{\alpha_{p_{i}}^{2}}>0
$$

Hence, because generator $p_{i}$ 's problem is guaranteed to have a global maximum, the profit-maximizing quantities of electricity and AS that generator $p_{i}$ sells into the spot markets can be determined.

On the other hand, retailer $r_{j}$ has little choice in selecting $X_{r_{i}}$ since it must meet the demand in its franchise area, $X_{r_{j}}^{T}$. This implies that $X_{r_{j}}^{*}=X_{r_{j}}^{T}$. Similarly, for the ISO, $Y_{I}^{*}=Y_{I}^{T} \equiv \gamma X^{T}$ since it's required to ensure that the specified levels of AS are acquired.

We can now use Equations 4 and 5 together with the retailers' and ISO's purchase requirements to evaluate the equilibrium prices that ensure both markets clear. The market-clearing conditions can, thus, be expressed as follows:

$$
\sum_{i=1}^{n} X_{p_{i}}^{*}=\sum_{j=1}^{m} X_{r_{j}}^{*}
$$

and

$$
\sum_{i=1}^{n} Y_{p_{i}}^{*}=Y_{I}^{*}
$$

Substituting Equation 4 and the retailers' purchase requirements into Equation 9, we obtain the equilibrium spot market price for electricity:

$$
P_{X}^{*}=\frac{\theta_{\ell}+\theta_{f}}{\alpha} X^{T}+\frac{\theta_{\ell}+f \theta_{f}}{\alpha} \gamma X^{T}
$$

where $\alpha \equiv \sum_{i=1}^{n} \alpha_{p_{i}}$. Similarly, by using Equation 5 and the ISO's purchase requirements together with Equation 10, we obtain the equilibrium spot market price for AS:

$$
P_{Y}^{*}=\frac{\theta_{\ell}(1-f)}{\alpha} X^{T}+\frac{\theta_{\ell}(1-f)}{\alpha} \gamma X^{T}
$$

The details of these derivations are left for the Appendix. Here, some of the intuitive properties of the spot prices are discussed. Looking first at Equation 11, the spot price of electricity consists of two terms. The first is simply the pro-rated cost of meeting the overall electricity demand, and the second term reflects the pro-rated cost of AS that are actually called upon to generate. Taken together, these two terms imply that the spot price of electricity fully compensates generators for the cost of all electricity that is produced. By contrast, the spot price of AS reflects opportunity costs. The first term in Equation 12 is a payment to generators for the pro-rated foregone revenue from electricity 
sales due to being on call for AS. Similarly, the second term compensates generators for the labor costs of operating capacity on call as AS not yet called upon to generate. Hence, even though the generator may not be required to produce electricity when on call for AS, it is, nevertheless, compensated because it missed out on the opportunity to use its productive capacity for other lucrative endeavors.

At this point, the generators' equilibrium sales of electricity and AS can be explicitly evaluated by substituting Equations 11 and 12 into Equations 4 and 5 , then solving simultaneously. While this approach would certainly yield the desired results, some effort can be avoided by using some intuition about the nature of perfectly competitive markets. Since it was assumed that all generators are identical and both electricity and AS requirements are fixed at $X^{T}$ and $\gamma X^{T}$, respectively, in equilibrium each generator will sell its pro-rated share of the overall requirements into each market. This then leads to the following:

Conjecture $1 X_{p_{i}}^{*}=\frac{\alpha_{p_{i}}}{\alpha} X^{T} \forall p_{i}$.

Conjecture $2 Y_{p_{i}}^{*}=\frac{\alpha_{p_{i}}}{\alpha} \gamma X^{T} \forall p_{i}$.

Verification of these is left for the Appendix.

\section{Empirical Analysis}

Using California market data, we can empirically test the hypothesis developed in Section 3 , viz., $P_{X}^{*}-P_{Y}^{*}=$ $\frac{\theta_{f}(1+f \gamma)}{\alpha} X^{T}+\frac{\theta_{\ell}(1+\gamma) f}{\alpha} X^{T}$. Equivalently, we can express this as:

$$
P_{X}^{*}=P_{Y}^{*}+\phi X^{T}
$$

Intuitively, this says that the spot price of electricity is equal to the spot price of AS plus:

1. the pro-rated incremental fuel cost of producing both electricity and AS that are called, and

2. the pro-rated incremental labor cost of producing AS that are called.

It is this hypothesis, i.e., Equation 13, that we will test using data available from the California markets.

Before proceeding with the analysis, however, some speculation is required as to which California markets are closest to the ideal markets in our perfectly competitive model. For the AS, the spinning reserve hourahead market is used, and for the electricity spot market, the ex-post supplemental energy prices and quantities are used. Hourly data for one year (1 June 1999 to 31 May 2000) obtained from the CAISO are analyzed.
During some hours, trading in the ex-post supplemental energy market did not occur, so only those hours with available data for both markets are used in the analysis. In order to be consistent, only the four regions that are common to both markets in question are analyzed. Finally, we take into account the fact that the CAISO had imposed various levels of price caps on all of its markets during the time period being studied. In 1999 , price caps were set at $\$ 250 / \mathrm{MW}$ until they were raised to $\$ 750 / \mathrm{MW}$ in October. In order to simplify the analysis, we discard all data points for which the ex-post supplemental energy price was strictly greater than $\$ 250 / \mathrm{MW}$. In terms of both number of data points and volume of energy traded, only a fraction of the data is discarded (almost $99 \%$ of the data points are retained or $98 \%$ of the energy volume is retained).

In order to test Equation 13, we cannot simply construct the following linear regression model and perform an ordinary least squares (OLS) regression:

$$
P_{i}=a+b X_{i}+\mu_{i}
$$

Here, $P_{i}$ refers to the month $i$ average energy price, $X_{i}$ is the month $i$ cumulative energy volume, and $\mu_{i}$ is a disturbance term that should be independent of $X_{i}$. However, because both the energy price and quantity are endogenous to the model, OLS estimators for $a$ and $b$ will likely be biased. In order to avoid this bias, we use the two-stage least squares (TSLS) regression procedure instead of OLS. For this, we need to specify some exogenous variables that are related to the endogenous ones, but not to the disturbances. We elect to use average monthly temperature ( $T_{i}$ for month $\left.i\right)$ and cumulative monthly precipitation $\left(R_{i}\right)$ as the exogenous variables. ${ }^{3}$ The structural model is then:

$$
X_{i}=\beta_{0}+\beta_{1} P_{i}+\mu_{i}
$$

$$
X_{i}=\eta_{0}+\eta_{1} P_{i}+\eta_{2} T_{i}+\eta_{3} R_{i}+\nu_{i}
$$

Equation 15 can be thought of as the "supply" expression, and Equation 16 can be considered the "demand" expression. The reduced form of this system is readily obtained by solving the structural model equations simultaneously:

$$
P_{i}=\pi_{10}+\pi_{11} T_{i}+\pi_{12} R_{i}+\epsilon_{1 i}
$$

\footnotetext{
${ }^{3}$ We obtain these data from the Western Regional Climate Center (http://нна.нrсc.dri.edu). For each CAISO zone, we selected a weather station in the most populous area. For Humboldt, it's Eureka (station 042910): for NP15, it's Sacramento (station 047633); for San Francisco (SF), it's SFO (station 047769); and for SP15, it's Los Angeles (station 045115).
} 


$$
X_{i}=\pi_{20}+\pi_{21} T_{i}+\pi_{22} R_{i}+\epsilon_{2 i}
$$

The $\pi$ terms are functions of the $\beta$ and $\eta$ terms from the structural model, and the $\epsilon$ terms are functions of all the terms involving $\beta, \eta, \mu$, and $\nu$. The TSLS procedure is as follows:

1. Regress $P_{i}$ on $T_{i}$ and $R_{i}$ to obtain the OLS estimators $\widehat{\pi_{10}}, \widehat{\pi_{11}}$, and $\widehat{\pi_{12}}$. Use these to obtain the prediction $\hat{P}_{i}=\widehat{\pi_{10}}+\widehat{\pi_{11}} T_{i}+\widehat{\pi_{12}} R_{i}$.

2. Estimate the structural model for the demand side using the following OLS regression:

$$
X_{i}=\beta_{0}+\beta_{1} \hat{P}_{i}+\mu_{i}
$$

By regressing the monthly cumulative ex-post supplemental energy volumes on the predicted monthly average ex-post supplemental energy prices, we obtain the OLS estimators $\hat{\beta}_{0}$ and $\hat{\beta}_{1}$. If our hypothesis in Equation 13 is correct, then we would expect the average spinning reserve spot price to be equal to $-\hat{\beta}_{0} / \hat{\beta}_{1}$, and $\hat{\beta}_{1}$ to be equal to $1 / \phi$, a large, positive number reflecting the fact that as the electricity spot price increases by a dollar to compensate the generator for its incremental labor and fuel expenses, additional MWs of capacity are made available for trade in the electricity spot market.

Table 1. Regression of Spot Market Quantities on Predicted Prices for All Zones (12 observations)

\begin{tabular}{|l|c|}
\hline Estimator & Value \\
\hline$\hat{\beta}_{0}$ & $-1.56 \times 10^{5} \mathrm{MW}$ \\
& $(-0.172)$ \\
$\hat{\beta}_{1}$ & $6.30 \times 10^{3} \mathrm{MW}^{2} / \$$ \\
& $(0.240)$ \\
\hline implied $P_{Y}$ & $\$ 24.64 / \mathrm{MW}$ \\
$R^{2}$ & 0.001 \\
\hline
\end{tabular}

Table 2. Regression of Spot Market Quantities on Predicted Prices for Humboldt (12 observations)

\begin{tabular}{|l|c|}
\hline Estimator & Value \\
\hline$\hat{\beta}_{0}$ & $-1.38 \times 10^{4} \mathrm{MW}$ \\
& $(-1.78)$ \\
$\hat{\beta}_{1}$ & $403.07 \mathrm{MW}^{2} / \$$ \\
& $(1.83)$ \\
\hline implied $P_{Y}$ & $\$ 34.27 / \mathrm{MW}$ \\
$R^{2}$ & 0.250 \\
\hline
\end{tabular}

Table 3. Regression of Spot Market Quantities on Predicted Prices for NP15 (12 observations)

\begin{tabular}{|l|c|}
\hline Estimator & Value \\
\hline$\hat{\beta}_{0}$ & $-4.48 \times 10^{5} \mathrm{MW}$ \\
& $(-1.39)$ \\
$\hat{\beta}_{1}$ & $1.84 \times 10^{4} \mathrm{MW}^{2} / \$$ \\
& $(2.01)$ \\
\hline implied $P_{Y}$ & $\$ 24.33 / \mathrm{MW}$ \\
$R^{2}$ & 0.288 \\
\hline
\end{tabular}

Table 4. Regression of Spot Market Quantities on Predicted Prices for SF (12 observations)

\begin{tabular}{|l|c|}
\hline Estimator & Value \\
\hline$\hat{\beta}_{0}$ & $-6.91 \times 10^{3} \mathrm{MW}$ \\
& $(-0.823)$ \\
$\hat{\beta}_{1}$ & $274.59 \mathrm{MW}^{2} / \$$ \\
& $(1.16)$ \\
\hline implied $P_{Y}$ & $\$ 25.15 / \mathrm{MW}$ \\
$R^{2}$ & 0.119 \\
\hline
\end{tabular}

In Table 1, the results of the TSLS regression over all included California zones are presented (the $t$ statistics appear in parentheses). While the coefficient estimators have the expected sign and magnitude (thereby resulting in a reasonable implied value for $P_{Y}$, the average spinning reserve spot price), the $R^{2}$ value is small and the estimators are statistically insignificant at the $10 \%$ level. Seeking a better fit, we perform the TSLS regression for each of the four main zones separately. We find that for San Francisco and SP15, the TSLS results are also not statistically significant (see Tables 4 and 5). For Humboldt, the results (see Table 2) are somewhat encouraging as the statistically significant coefficient estimators are of the desired sign, but the implied $P_{Y}$ value is not close at all to the actual average spinning reserve day-ahead prices in the zone. For

Table 5. Regression of Spot Market Quantities on Predicted Prices for SP15 (12 observations)

\begin{tabular}{|l|c|}
\hline Estimator & Value \\
\hline$\hat{\beta}_{0}$ & $5.04 \times 10^{4} \mathrm{MW}$ \\
& $(0.139)$ \\
$\hat{\beta}_{1}$ & $-238.04 \mathrm{MW}^{2} / \$$ \\
& $(-0.021)$ \\
\hline implied $P_{Y}$ & $\$ 211.91 / \mathrm{MW}$ \\
$R^{2}$ & $4.48 \times 10^{-5}$ \\
\hline
\end{tabular}


Table 6. Spinning Reserve Hour-Ahead Prices By Zone ( 34975 observations)

\begin{tabular}{|l|c|c|}
\hline Zone & Mean $(\$ /$ MW) & S. D. $(\$ / M W)$ \\
\hline All & 12.42 & 36.78 \\
Humboldt & 0.87 & 7.14 \\
NP15 & 23.28 & 52.35 \\
San Francisco & 4.77 & 16.30 \\
SP15 & 20.77 & 44.44 \\
\hline
\end{tabular}

zone NP15, however, the results (see Table 3 ) tend to support the hypothesis. Here, the coefficients are of the desired sign and magnitude, and they are statistically significant. The resulting implied $P_{Y}$ value is quite close to the actual average spinning reserve day-ahead prices for the zone (see Table 6). Moreover, the $R^{2}$ value is higher at $28.8 \%$. These results provide limited, but encouraging, empirical support of our spot pricing hypothesis. Indeed, our model is able to explain over one quarter of the variation in the spot pricing of electricity in spite of the problems observed in California markets. We can reasonably concentrate on the results from zone NP15 because it accounted for $81.2 \%$ of all spot market electricity volume traded. The fact that our model is unable to explain much of the variation in spot pricing in the relatively smaller zones implies that competitive electricity trading is unlikely to flourish there. Intuitively, this is what we would expect, i.e., smaller trading zones are less competitive than larger ones.

\section{Conclusions}

In this paper, a theoretical treatment of spot pricing of electricity and AS as alternate products in a perfectly competitive environment is developed and applied to California data. In outlining the changes effected by deregulation, we note that the problem of managing system reliability in a competitive market may be exacerbated if there is no market-based methodology for pricing AS. In order to assess equilibrium spot prices for electricity and AS, a perfectly competitive model with generators, retailers, and an ISO is established with trading rules loosely based on California conditions. The fundamental physical and financial links between the electricity and AS markets are reflected in this integrated model. By assuming that each market agent acts out of self interest in order to maximize its wealth and that all markets clear, we arrive at the equilibrium spot prices for electricity and AS. We show that the AS spot price reflects the opportunity costs of not using productive generating capacity for the other profitable endeavor.

In order to test empirically the validity of the model, a TSLS analysis of the relationship between the spot prices of electricity and AS is performed. In the largest zone of California, the model explains a significant proportion of the variation in the spot price of electricity (with respect to the AS spot price). The analysis is simplified by the absence of real world phenomena such as transmission constraints, market power, and multiple types of AS. However, keeping the model simple allows formalization of the relationship between electricity and AS spot pricing. Enriching the model by incorporating these aforementioned realities will be part of future research. In the meantime, we expect that with a market-based methodology for pricing AS, no matter how crude, both the ISO and market agents alike will be able to determine the market value of AS in terms of the spot price of electricity.

\section{References}

[1] Joskow, P. L. (1998), "Restructuring, Competition, and Regulatory Reform in the U.S. Electricity Sector," in Designing Competitive Electricity Markets, edited by H.P. Chao and H.G. Huntington, Kluwer Academic Publishers, MA.

[2] California Independent System Operator (1999), "Annual Report on Market Issues and Performance," Corporate Report, CAISO.

[3] Gómez, T. , C. Marnay, A. Siddiqui, L. Liew, and M. Khavkin (1999), "Ancillary Services Markets in California," Ernest Orlando Lawrence Berkeley National Laboratory Report LBNL-43986, Berkeley, CA.

[4] Wolfram, C. D. (1999), "Measuring Duopoly Power in the British Electricity Spot Market," American Economic Review, 89: 805-826.

[5] Borenstein, S. and J. Bushnell (1998), "An Empirical Analysis of the Potential for Market Power in California's Electricity Industry," POWER Working Paper PWP044r, University of California Energy Institute, CA.

[6] Borenstein, S., J. Bushnell, and C.R. Knittel (1999), "Market Power in Electricity Markets: Beyond Concentration Mea- 
sures," POWER Working Paper PWP059r, University of California Energy Institute, CA.

$[7]$

Borenstein, S. , J. Bushnell, and F. Wolak (1999), "Diagnosing Market Power in California's Deregulated Wholesale Electricity Market," POWER Working Paper PWP064, University of California Energy Institute, CA.

[8] Siddiqui, A. S. , C. Marnay, and M. Khavkin (2000), "Excessive Price Volatility in the California Ancillary Services Markets: Causes, Effects, and Solutions," Electricity Journal, 13(6): 58-68.

[9] Bessembinder, H. , M.L. Lemmon (1999), "Equilibrium Pricing and Optimal Hedging in Electricity Forward Markets," Proceedings of the Fourth Annual POWER Research Conference, Berkeley, CA.

\section{Appendix}

Substituting Equation 4 and the retailers' purchase requirements into Equation 9, we obtain:

$$
\begin{gathered}
\sum_{i=1}^{n} \frac{\alpha_{p_{i}} P_{X}-\left(\theta_{\ell}+f \theta_{f}\right) Y_{p_{i}}^{*}}{\theta_{\ell}+\theta_{f}}=\sum_{j=1}^{m} X_{r_{j}}^{T} \\
\Rightarrow \frac{\alpha}{\theta_{\ell}+\theta_{f}} P_{X}-\frac{\left(\theta_{\ell}+f \theta_{f}\right)}{\theta_{\ell}+\theta_{f}} \sum_{i=1}^{n} Y_{p_{i}}^{*}=X^{T}
\end{gathered}
$$

Making use of Equation 10:

$$
\begin{gathered}
\Rightarrow \frac{\alpha}{\theta_{\ell}+\theta_{f}} P_{X}-\frac{\left(\theta_{\ell}+f \theta_{f}\right)}{\theta_{\ell}+\theta_{f}} Y_{I}^{*}=X^{T} \\
\Rightarrow \frac{\alpha}{\theta_{\ell}+\theta_{f}} P_{X}-\frac{\left(\theta_{\ell}+f \theta_{f}\right)}{\theta_{\ell}+\theta_{f}} \gamma X^{T}=X^{T} \\
\Rightarrow \frac{\alpha}{\theta_{\ell}+\theta_{f}} P_{X}=\left[1+\frac{\left(\theta_{\ell}+f \theta_{f}\right) \gamma}{\theta_{\ell}+\theta_{f}}\right] X^{T} \\
\Rightarrow \frac{\alpha}{\theta_{\ell}+\theta_{f}} P_{X}=\left[\frac{\theta_{\ell}+\theta_{f}+\left(\theta_{\ell}+f \theta_{f}\right) \gamma}{\theta_{\ell}+\theta_{f}}\right] X^{T} \\
\Rightarrow P_{X}=\left[\frac{\theta_{\ell}(1+\gamma)+\theta_{f}(1+f \gamma)}{\alpha}\right] X^{T}
\end{gathered}
$$

This is equivalent to Equation 11.

In order to derive Equation 12, substitute Equation 5 and the ISO's purchase requirements into Equation 10:

$$
\sum_{i=1}^{n} \frac{\alpha_{p_{i}}\left(P_{Y}+f P_{X}\right)-\left(\theta_{\ell}+f \theta_{f}\right) X_{p_{i}}^{*}}{\theta_{\ell}+f^{2} \theta_{f}}=Y_{I}
$$

$\Rightarrow \frac{\alpha}{\theta_{\ell}+f^{2} \theta_{f}}\left(P_{Y}+f P_{X}\right)-\frac{\left(\theta_{\ell}+f \theta_{f}\right)}{\theta_{\ell}+f^{2} \theta_{f}} \sum_{i=1}^{n} X_{p_{i}}^{*}=\gamma X^{T}$

Making use of Equation 9:

$$
\begin{gathered}
\Rightarrow \frac{\alpha}{\theta_{\ell}+f^{2} \theta_{f}}\left(P_{Y}+f P_{X}\right)-\frac{\left(\theta_{\ell}+f \theta_{f}\right)}{\theta_{\ell}+f^{2} \theta_{f}} \sum_{j=1}^{m} X_{r_{j}}^{*}=\gamma X^{T} \\
\Rightarrow \frac{\alpha}{\theta_{\ell}+f^{2} \theta_{f}}\left(P_{Y}+f P_{X}\right)-\frac{\left(\theta_{\ell}+f \theta_{f}\right)}{\theta_{\ell}+f^{2} \theta_{f}} \sum_{j=1}^{m} X_{r_{j}}^{T}=\gamma X^{T} \\
\Rightarrow \frac{\alpha}{\theta_{\ell}+f^{2} \theta_{f}}\left(P_{Y}+f P_{X}\right)-\frac{\left(\theta_{\ell}+f \theta_{f}\right)}{\theta_{\ell}+f^{2} \theta_{f}} X^{T}=\gamma X^{T} \\
\Rightarrow \frac{\alpha}{\theta_{\ell}+f^{2} \theta_{f}}\left(P_{Y}+f P_{X}\right)=\left[\gamma+\frac{\left(\theta_{\ell}+f \theta_{f}\right)}{\theta_{\ell}+f^{2} \theta_{f}}\right] X^{T} \\
\Rightarrow \frac{\alpha}{\theta_{\ell}+f^{2} \theta_{f}} P_{Y}=\left[\frac{\gamma\left(\theta_{\ell}+f^{2} \theta_{f}\right)}{\theta_{\ell}+f^{2} \theta_{f}}\right] X^{T} \\
+\left[\frac{\left(\theta_{\ell}+f \theta_{f}\right)}{\theta_{\ell}+f^{2} \theta_{f}}\right] X^{T} \\
-\frac{\alpha}{\theta_{\ell}+f^{2} \theta_{f}} f P_{X} \\
\Rightarrow P_{Y}=\left[\frac{\theta_{\ell}(1+\gamma)+f \theta_{f}(1+f \gamma)}{\alpha}\right] X^{T}-f P_{X}
\end{gathered}
$$

Now by substituting in Equation 11, we obtain:

$$
\begin{aligned}
P_{Y}= & {\left[\frac{\theta_{\ell}(1+\gamma)+f \theta_{f}(1+f \gamma)}{\alpha}\right] X^{T} } \\
& -\left[\frac{f \theta_{\ell}(1+\gamma)+f \theta_{f}(1+f \gamma)}{\alpha}\right] X^{T} \\
\Rightarrow & P_{Y}=\left[\frac{\theta_{\ell}(1+\gamma)(1-f)}{\alpha}\right] X^{T}
\end{aligned}
$$

This is equivalent to Equation 12.

By substituting Conjecture 2 and Equation 11 into Equation 4, we can affirm Conjecture 1:

$$
\begin{aligned}
\Rightarrow X_{p_{i}}^{*}= & {\left[\frac{\alpha_{p_{i}}\left(\theta_{\ell}(1+\gamma)+\theta_{f}(1+f \gamma)\right)}{\alpha\left(\theta_{\ell}+\theta_{f}\right)}\right] X^{T} } \\
& -\left[\frac{\alpha_{p_{i}} \gamma\left(\theta_{\ell}+f \theta_{f}\right)}{\alpha\left(\theta_{\ell}+\theta_{f}\right)}\right] X^{T} \\
\Rightarrow X_{p_{i}}^{*}= & {\left[\frac{\alpha_{p_{i}}\left(\theta_{\ell}+\gamma \theta_{\ell}+\theta_{f}+f \gamma \theta_{f}\right)}{\alpha\left(\theta_{\ell}+\theta_{f}\right)}\right] X^{T} } \\
& \left.-\left[\frac{\alpha_{p_{i}}\left(\gamma \theta_{\ell}+f \gamma \theta_{f}\right)}{\alpha\left(\theta_{\ell}+\theta_{f}\right)}\right] X^{T}\right] \\
\Rightarrow X_{p_{i}}^{*}= & {\left[\frac{\alpha_{p_{i}}\left(\theta_{\ell}+\theta_{f}\right)}{\alpha\left(\theta_{\ell}+\theta_{f}\right)}\right] X^{T} } \\
\Rightarrow X_{p_{i}}^{*}= & \frac{\alpha_{p_{i}}}{\alpha} X^{T}
\end{aligned}
$$


Similarly, by substituting Conjecture 1 and Equation 12 into Equation 5, we affirm Conjecture 2:

$$
\begin{aligned}
\Rightarrow Y_{p_{i}}^{*}= & {\left[\frac{\alpha_{p_{i}}\left(\theta_{\ell}(1+\gamma)(1-f)\right)}{\alpha\left(\theta_{\ell}+f^{2} \theta_{f}\right)}\right] X^{T} } \\
& +\left[\frac{\alpha_{p_{i}}\left(f \theta_{\ell}(1+\gamma)\right)}{\alpha\left(\theta_{\ell}+f^{2} \theta_{f}\right)}\right] X^{T} \\
& +\left[\frac{\alpha_{p_{i}} f \theta_{f}(1+f \gamma)}{\alpha\left(\theta_{\ell}+f^{2} \theta_{f}\right)}\right] X^{T} \\
& -\left[\frac{\alpha_{p_{i}}\left(\theta_{\ell}+f \theta_{f}\right)}{\alpha\left(\theta_{\ell}+f^{2} \theta_{f}\right)}\right] X^{T} \\
\Rightarrow Y_{p_{i}}^{*}= & {\left[\frac{\alpha_{p_{i}}\left(\theta_{\ell}+\gamma \theta_{\ell}-f \theta_{\ell}+f \theta_{\ell}\right)}{\alpha\left(\theta_{\ell}+f^{2} \theta_{f}\right)}\right] X^{T} } \\
& +\left[\frac{\alpha_{p_{i}}\left(f \gamma \theta_{\ell}-f \gamma \theta_{\ell}+f \theta_{f}\right)}{\alpha\left(\theta_{\ell}+f^{2} \theta_{f}\right)}\right] X^{T} \\
& +\left[\frac{\alpha_{p_{i}}\left(f^{2} \gamma \theta_{f}-\theta_{\ell}-f \theta_{f}\right)}{\alpha\left(\theta_{\ell}+f^{2} \theta_{f}\right)}\right] X^{T} \\
\Rightarrow Y_{p_{i}}^{*}=\quad & {\left[\frac{\alpha_{p_{i}}\left(\gamma \theta_{\ell}+f^{2} \gamma \theta_{f}\right)}{\alpha\left(\theta_{\ell}+f^{2} \theta_{f}\right)}\right] X^{T} } \\
\Rightarrow Y_{p_{i}}^{*}=\quad & \frac{\alpha_{p_{i}}\left(\theta_{\ell}+f^{2} \theta_{f}\right)}{\alpha\left(\theta_{\ell}+f^{2} \theta_{f}\right)} \gamma X^{T} \\
\Rightarrow Y_{p_{i}}^{*}=\quad & \frac{\alpha_{p_{i}} \gamma X^{T}}{\alpha}
\end{aligned}
$$

